\title{
Eric T. Meyer \& Ralph Schroeder (2015) Knowledge Machines: Digital Transformations of the Sciences and Humanities. Cambridge, MA: The MIT Press.
}

\author{
Dominique Vinck \\ dominique.vinck@unil.ch
}

Knowing machines analyses the transformations taking place in the sciences owing to the use of digital technologies. It focuses especially on the collaborative use of digital tools in different disciplines, ranging from high-energy physics and biomedical sciences to the digital humanities and social sciences. It questions the transformations at work in the organisation and practices of research, in the relations between researchers and their public, but also in knowledge content. As they explore the way in which digital tools reconfigure knowledge production, the authors, Eric Meyer and Ralph Schroeder from the Oxford Internet Institute, question whether the distributed and collaborative use of digital tools and data are creating new openings for research.

The book targets researchers from all disciplines, from the physical sciences to the human and social sciences, insofar as they are all affected by digital tools. It also targets the information technology and science researchers, engineers and policymakers who support and guide the developments taking place. The various social groups, increasingly concerned by the production of knowledge, must not be forgotten either given the growing role they are being asked to play via digital tools and open data.

Eric Meyer and Ralph Schroeder study collaborative e-research and strive to qualify the collaboration made possible by digital tools. Thanks to institutional enthusiasm for research cyber-infrastructures but also to the initiatives of scientific communities, many developments have seen the light of day. The authors explore a series of thought-provoking cases, which are very well described and documented in the book: the networking of computers to provide enough calculation capacity to process the masses of data generated by high energy physics experiments (Grid Particle Physics); the involvement of countless non-specialist astrophysics enthusiasts (crowd-sourcing) to qualify photographs of galaxies (Galaxy Zoo) and hence drive the algorithms (machine learning), which will perform the same task automatically in the future; the pooling and processing of experimental data produced by many research groups in the field of medicine and genetics (GAIN); the collection of data about the entire Swedish population, which supposes public's trust; the sharing of digital photos of whale fins so as to monitor and study their population across the entire world and in the very long term (SPLASH); the involvement of amateur critics to interpret, annotate and discuss all the passages of a writer's work (Pynchon Wiki).

These case studies allow the authors to address a series of relevant topics: the conditions for sharing and circulating data; trust in research institutions; the building of compatibility of concepts, methods and instruments; the creation of a fair public-private partnership; the sustainable financing of research infrastructures (cf. Kleiner et al., 2013) and the conditions required for upholding collaboration; recognition 
mechanisms; and the transformation of relations between professional researchers and other actors in society.

Following an introduction, the book comprises of nine chapters. It starts with a focus on the conceptual framework and then provides a general description of the emergence of digital technology in research (financing, publication, visibility). These chapters are then followed by four others in which the authors present some very good case studies. The last three chapters offer a discussion of open science, the limits of digital collaborative research and a comparative reading of knowledge machines.

Eric Meyer and Ralph Schroeder draw inspiration from the work of lan Hacking in order to identify "styles of science" as well as from actornetwork theory to characterise the sociotechnical networks corresponding to each project examined. In the last chapter, the cases can be compared based on a model. This conceptual framework, as well as the references to Randall Collins, Richard Whitley, Terry Shinn and Bernward Joerges, allows the authors to qualify the role played by digital tools in each case. They question whether these tools channel the scientification of the sciences, whether they encourage the emergence of a consensus between researchers by conferring greater objectivity on the phenomena studied and whether they lead to the transfer of tools, methods and forms of work organisation between disciplines (e.g. crowdsourcing, computer networking, data circulation). Using Richard Whitley's organisational characterisation of scientific disciplines in relation to their degree of strategy/functional dependence, they question whether digital tools are easier to embed in certain configurations rather than others. Then, in order to build more global analyses of the phenomenon, they call on Rob Kling's work on the development of information systems, focusing especially on uses and routines, as well as the notion of the computerisation movement (taking into account public communication on technologies) and Scott Frickel and Neil Gross's notion of the intellectual and scientific movement (the dominant approach to a problem). The authors query whether e-research corresponds more to a computerisation movement (demonstration of the advantages offered by digital tools) or to an intellectual and scientific movement (thinking on how to improve scientific approaches and practices). Eric Meyer and Ralph Schroeder claim to go beyond science and technology studies, which, according to them, are limited to case studies.

The problems pinpointed by the authors at the outset and the case studies discussed are of the highest interest. In the last chapters, they offer a comparative and overall interpretation of the situation. When it comes to open research data, they move away from the case studies and refer to Robert Merton's ethos of science, the pressures behind the promotion and criticism of free and open access to data and the problem of limited attention space. However, their thinking here does not return to the case studies. Similarly, rather than deepening the analysis of the cases described earlier in the book, the penultimate chapter on the limits of digital collaborative research introduces new cases. This chapter deals with the failure of a mapping tool owing to institutional and legal barriers. The authors also turn their attention to web archives, questioning their future, which they portray as potentially gathering dust, like any other archive when not used. They next launch into some general reflections about the limits of data sharing, the ethical challenges, and the questions of trust and economic requirements.

The last chapter, on the other hand, returns to the case studies as the authors model and compare these in order to draw some conclusions about the role of technologies in the transformation of sciences. The authors nevertheless underline that the outcome of this movement is still unsure, and that it depends on the disciplines. The transformations explored are not revolutionary.

\section{References}

Brian Kleiner, Isabelle Renschler, Boris Wernli, Peter Farago, \& Dominique Joye (eds) (2013) Understanding Research Infrastructures in the Social Sciences. Zurich: Seismo 